\title{
A Novel Approach for Searching an Element in a Sorted Array - Log Search
}

\author{
Arpan Bairagi ${ }^{*}$, Rejoy Chakraborty ${ }^{1}$, Shree Mitra $^{1}$ and Soumya Paul ${ }^{2}$ \\ ${ }^{I}$ Department of Computer Science, Ramakrishna Mission Vivekananda Centenary College, Rahara, West Bengal, India \\ ${ }^{2}$ Professor, Department of Computer Science and Engineering, St. Mary's Technical Campus, Kolkata, India \\ *Corresponding author: arpan.bairagi123456789@gmail.com
}

\begin{abstract}
In computer science, various searching algorithms are available. We choose among them according to the situation. There are some efficient searching algorithms like Binary Search, Jump Search etc. In this paper a new searching algorithm which tracks the key element faster in some cases is proposed. This algorithm is Log Search. This algorithm only works for a sorted array. This algorithm searches the key element in a sub-array of the full array. If there is no possibility to find the key element, it will try to find out the next sub-array. If there is a possibility to find it to that sub-array, then it will recursively do the same thing.
\end{abstract}

Keywords: Searching algorithm, Logarithmic, Binary Search, Log Search

In our real life, searching is a common work to do. For example, finding a chapter name in a book, finding a particular file in a bunch of files is same to them. We use searching also in our computer system as searching a specific file throughout the directories, finding a word in a word file etc. Higher level applications are web searching, location finding in Google Maps, GPS tracker ${ }^{[1]}$ etc. As there are various searching algorithms in Computer Science, we propose an algorithm as searching algorithm. We describe the algorithm and analyzing the parameters of the algorithm like time complexity, space complexity, accuracy, robustness etc.

Traditional search algorithms are like linear search, binary search, interpolation search and jump search. There are some proposed search algorithms too, as network localization using tree search algorithm, a fast string searching algorithm etc. ${ }^{[2]}$. Some researchers also proposed some other searching algorithms: Ternary Search, Q Search ${ }^{[3]}$, O Search ${ }^{[4]}$ etc. RS Boyer et al. proposed a Fast String Searching Algorithm ${ }^{[5]}$. 


\section{Objective}

Here we shall take a sorted array. Our main aim is to search our desired key value from a sorted array efficiently. At first we should have some equality conditions to check whether there is our desired value or not. For more quick response we should add an equality condition in the mid index of array other than lowest and highest index. If there is no match we should break the array and search the element in sub arrays. So our main aim is to divide and search our desired element.

If there is no match in the last, we can conclude that the element is not present in the given sorted array.

\section{Methodology}

This method is applicable only for Sorted Array. If the size of the array is $n$ then we shall calculate the $\mathrm{n} / \log _{2} \mathrm{n}$ (say div). Basically here we will calculate $\mathrm{n}=(\mathrm{high}-\mathrm{low}+1)$ where high is the highest index and low is lowest index. First we will check, whether the value may exist in the range of the array or not. If in the range, then we will check whether the value is equal to array [low] or array [mid of array] or array [high]. If it is equal, then it will return the index. If it is not, then we will set the index high at (low + div). Now we will again check whether the value may exist in the range or not (i.e. in the new range). If it is in the range, then we will again check the equality condition, here length is the size of that sub array. Now, there are two possibilities, one is the key may in the range and another is the key definitely not in the range.

If it is not in the range, then we will set low $=$ high and high $=$ previous high $+\operatorname{div}+1$. If it is not in the range, then we will set low $=$ high and high $=$ previous high $+\operatorname{div}+1$. Now also, we will check the equality conditions for that range and if it is not equal then we will check the possibility of existence in the range. It will shift the index until a match of value or a possibility of existence in that range.

Now, whenever it finds the value exist in the range in the sub array then it recursively calls the searching function and again divides the sub array to its subsequent sub array until a match with the value the mentioned three indexes. At last, if there is no match it will indicate that the value is not in array.

Two points we have to observe that, high $>$ low and high $<=$ length of array always [ since high max $=$ length of array -1].

And whenever $\operatorname{div}=1$, then we will assign $\operatorname{div}=0$ for safety purpose.

\section{Proposed Heuristic}

In below, we are giving the pseudo code of our proposed searching algorithm.

$/ *$ high $<$ - last index of the array (or sub-array)

low $<$ - first index of the array (or sub-array)

arr $<-$ array

target $<$ - the value we want to search

$\operatorname{div}<$ - splitting value of the array

$\lg <-\log$ value base */ 
LogSearch (arr[], target, div, low, high) $\{* * *$ start of the LogSearch method $* * *$

if (length of arr $>=$ high and high $>=$ low) $\{* * * *(1)$ if $* * * *$

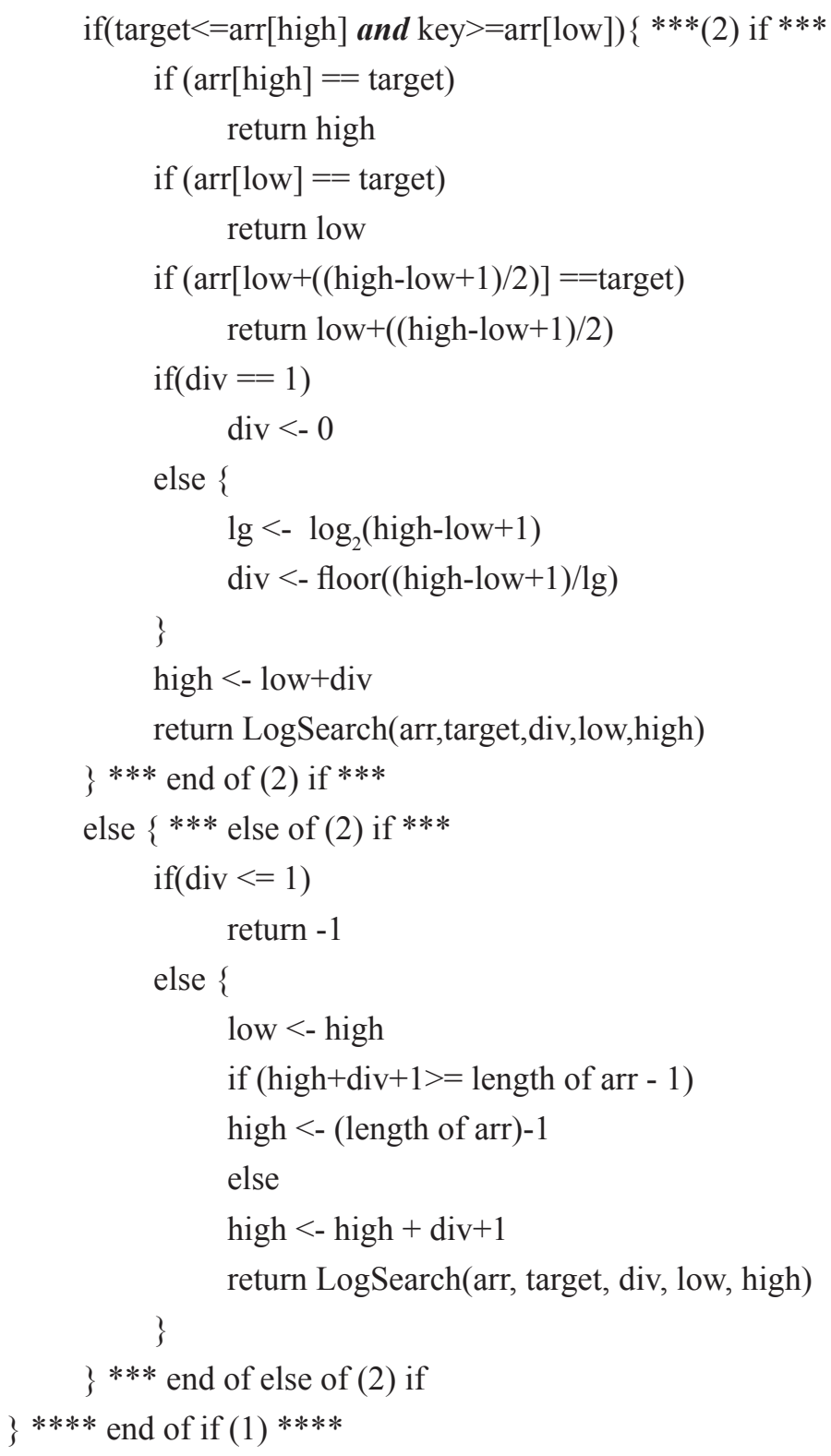

Note: Initially we have to pass 0 as the div value

Note: Here we have given the logic for sorted array in ascending order. It can be implemented for sorted arrays in descending orders also by just reversing the logic of range. 


\section{Example Illustration}

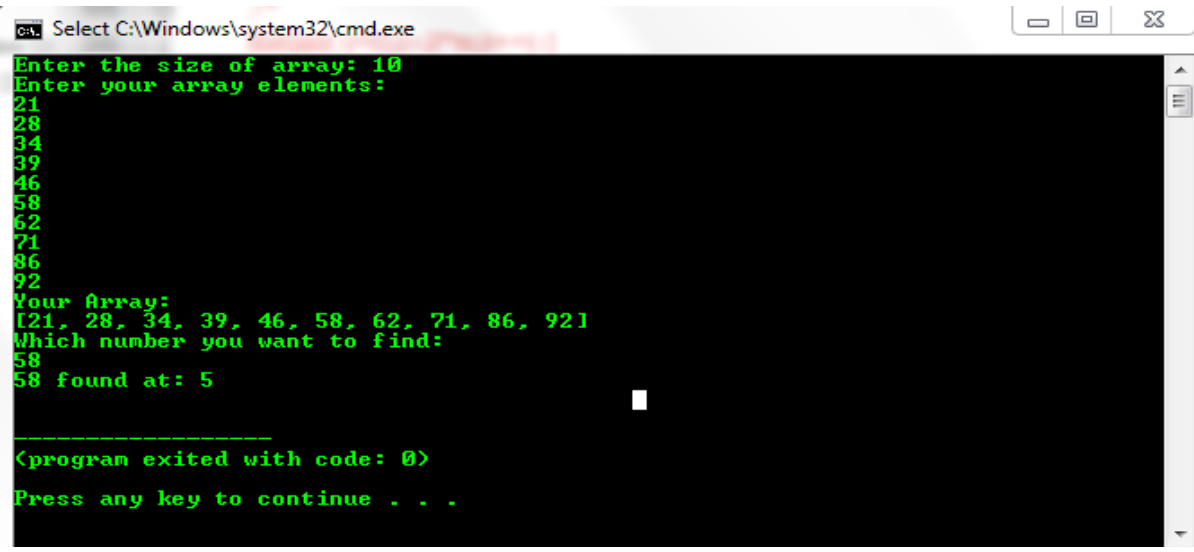

Fig. 1: A number is found from an arbitrary position

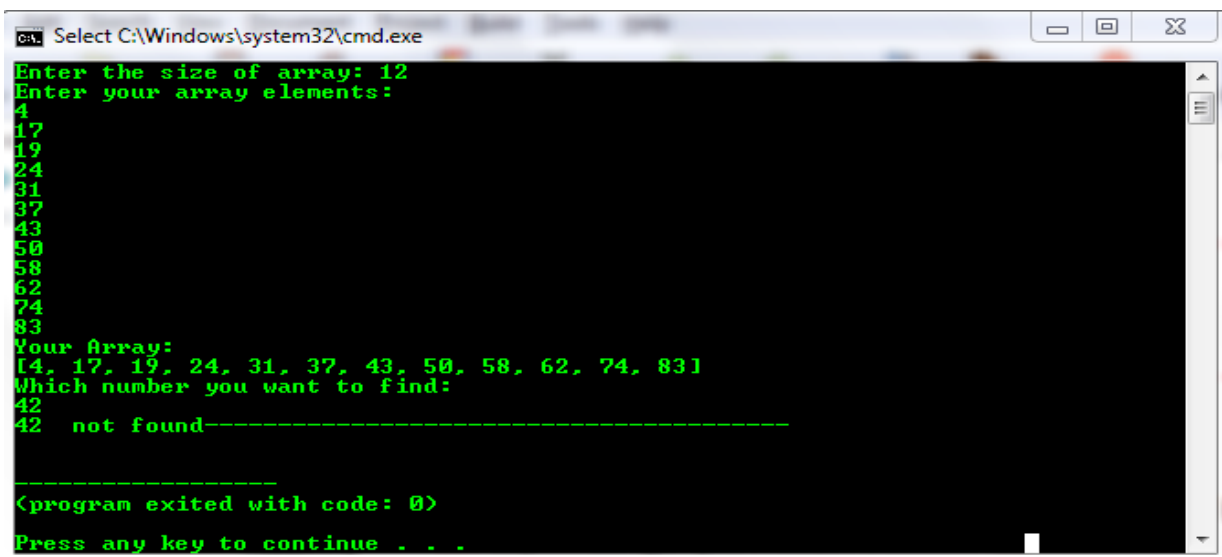

Fig. 2: A number is not found from an arbitrary position

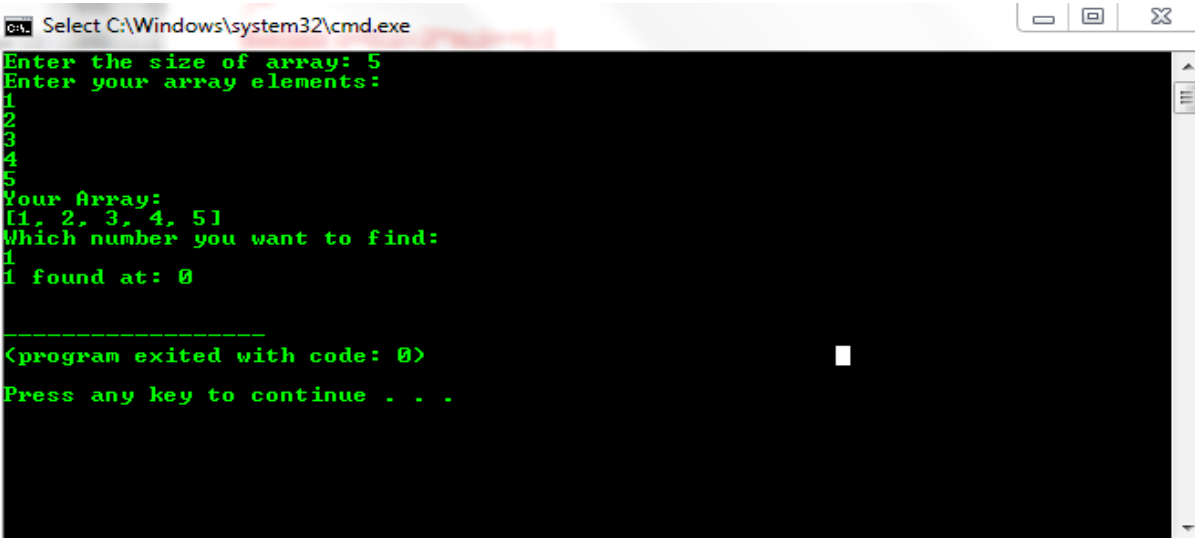

Fig. 3: First element is found 


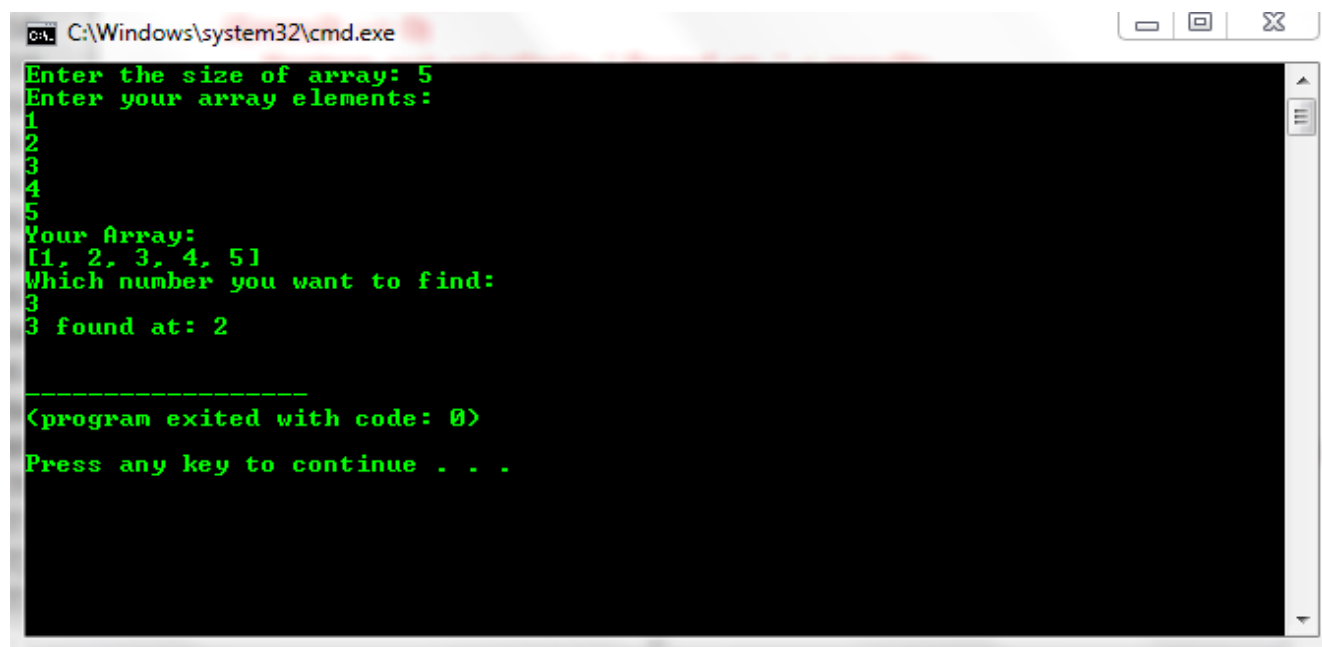

Fig 4: Mid element is found

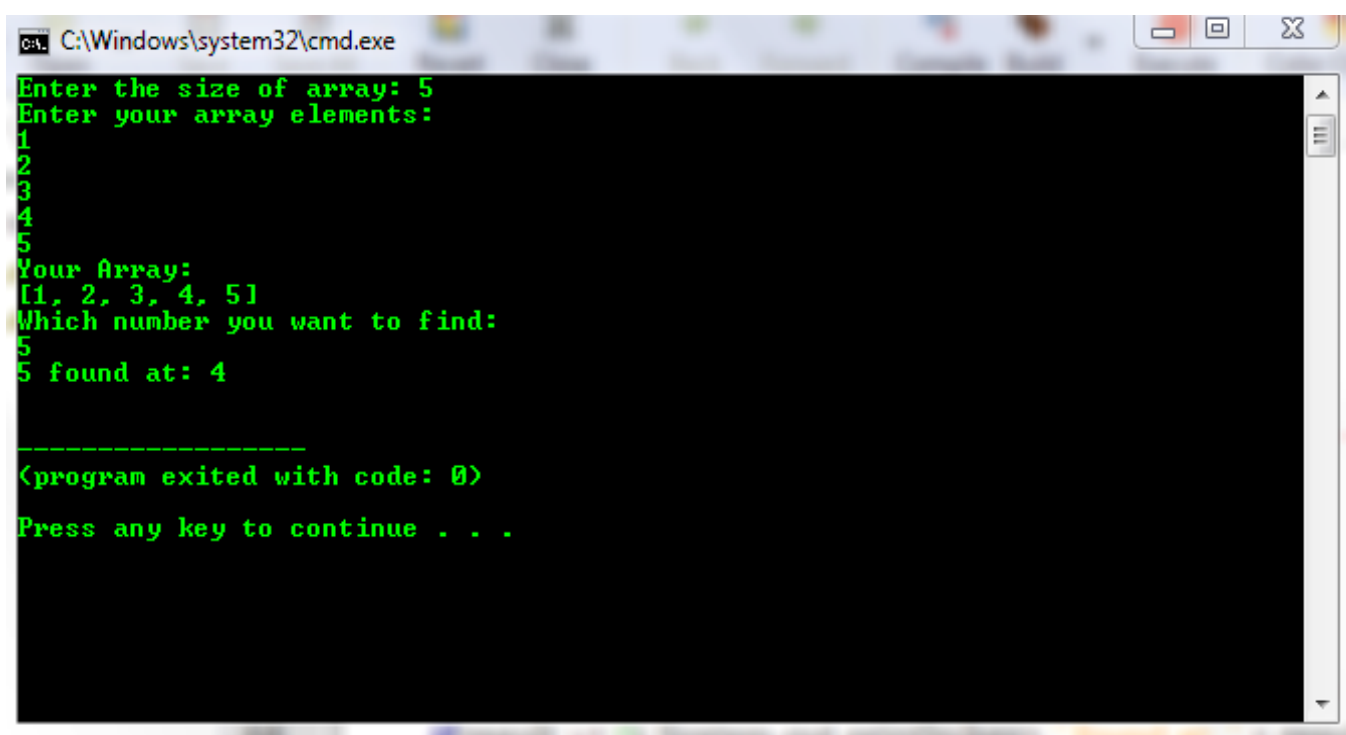

Fig. 5: Last element is found

\section{CONCLUSION}

In this paper presented a novel searching algorithm. This searching algorithm can be only applicable for sorted arrays. It checks some equality conditions to match the key value in first, mid and in last positions of the current working array. If there is no match, then it divides the array to its logarithmic length and recursively check the conditions and recursively follow the same divide and check method until there is a match. As this divides the array in its logarithmic value, it can be called as Log Search. 


\section{REFERENCES}

1. Chamikara, M.A.P., Yapa, Y.P.R.D., Kodituwakku, S.R. and Gunathilake, J. An Efficient Algorithm to Detect the Nearest Location of a Map for a Given Theme.

2. Sultana, N., Paira, S., Chandra, S. and Alam, S.S. 2017. A brief study and analysis of different searching algorithms. In 2017 Second International Conference on Electrical, Computer and Communication Technologies (ICECCT) (pp. 1-4). IEEE.

3. Taranjit Khokhar, September 2016, Q (Quaternary) Search Algorithm, IJIRT, 3(4): 143908.

4. Maheshwari, B.R. 2019. Octonary (O) Search Algorithm.

5. Boyer, R.S. and Moore, J.S. 1977. A fast string searching algorithm. Communications of the ACM, 20(10): 762-772.

6. Wu, S. and Manber, U. 1994. A fast algorithm for multi-pattern searching (pp. 1-11). University of Arizona. Department of Computer Science.

7. Aikio, J. and Mähönen, P. 1998. A simple void-searching algorithm. The Astrophysical Journal, 497(2): 534.

8. Aggarwal, A., Klawe, M.M., Moran, S., Shor, P. and Wilber, R. 1987. Geometric applications of a matrix-searching algorithm. Algorithmica, 2(1-4): 195-208.

9. Topsok, F. 2006. Some bounds for the logarithmic function. Inequality theory and applications, 4: 137.

10. Kenney, R. 2005. Students' understanding of logarithmic function notation. In annual meeting of the North American Chapter of the International Group for the Psychology of Mathematics Education, Hosted by Virginia Tech University Hotel Roanoke \& Conference Center, Roanoke, VA. Retrieved from http://www. allacademic. com/meta/p24727index. html.

11. Shneiderman, B. 1978. Jump searching: a fast sequential search technique. Communications of the $A C M, 21(10): 831-834$.

12. Cormen, T.H., Stein C., Rivest R.L. and Leiserson, C.E., Introduction to Algorithms, $3^{\text {rd }}$ Edition, The MIT Press. 